\title{
The Herschel InfraRed Galactic Plane Survey: A Panoramic View of Star Formation in Milky Way
}

\author{
Davide Elia* \\ INAF-Istituto di Astrofisica e Planetologia Spaziali, Via Fosso del Cavaliere 100, I-00133 Roma, \\ Italy \\ E-mail: davide.elialiaps.inaf.it
}

\section{Sergio Molinari}

INAF-Istituto di Astrofisica e Planetologia Spaziali, Via Fosso del Cavaliere 100, I-00133 Roma, Italy

\section{Eugenio Schisano}

INAF - Istituto di Radioastronomia, and Italian ALMA Regional Centre, Via Gobetti 101, I-40129 Bologna

\section{Adriano Baldeschi}

INAF-Istituto di Astrofisica e Planetologia Spaziali, Via Fosso del Cavaliere 100, I-00133 Roma, Italy

Hi-GAL, the Herschel unbiased survey of the entire Galactic plane (70-500 $\mu \mathrm{m})$, made available a vast mine of regions for studying early phases of star formation, especially in the high-mass regime, in different Galactic environments, providing an overall view of the Milky Way as a star formation engine. Here we present the first release of the Hi-GAL physical catalog, constituted by about 140000 entries, namely the compact sources detected by Herschel and showing a regular spectral energy distribution, eligible for grey-body fit. Complementary MSX, WISE, MIPSGAL, ATLASGAL and BGPS photometry was also used to extend the wavelength coverage. A large fraction of sources in the catalog is associated with a heliocentric distance estimate, which range from $<1 \mathrm{kpc}$ to $>15 \mathrm{kpc}$, so that our sample of compact sources spans more than two orders of magnitude of linear sizes, corresponding to different object classes (from cores to clumps and even small clouds). This allows us to study the behaviour of star-forming structures on different spatial scales. Furthermore, we use source properties as temperature, mass, surface density, bolometric luminosity to build an evolutionary picture, and to search for conditions for high-mass star formation to occur.

Frontier Research in Astrophysics - III (FRAPWS2018)

28 May - 2 June 2018

Mondello (Palermo), Italy

\footnotetext{
* Speaker.
} 


\section{Introduction}

The massive stars $\left(M \gtrsim 8 M_{\odot}\right)$ can exert a big influence on the evolution of their environment. The appearance of intermediate- and high-mass forming protostars is the event that drives the radiative energy budget in these systems. However, the formation of these objects is somehow elusive, due to the shorter time scales compared with Solar type stars, and to the fact that massive star formation is an intrinsically less frequent event. The evolutionary sequence for a young massive star is so fast that in most cases such an object reaches the zero-age main sequence before totally dissipating their parental cloud, so that observations in the infrared are requested to study the earliest phases of massive star formation.

The Herschel Infrared Galactic Plane Survey [1] is the keystone of a suite of continuum Milky Way surveys from the near-IR to the radio, and covers five wavebands at 70, 160, 250, 350 and $500 \mu \mathrm{m}$, encompassing the peak of the spectral energy distribution of cold dust for $8<T<50 \mathrm{~K}$. It gave a map of the dust distribution and temperature in the inner Galactic plane with an unprecedented spatial resolution and dynamic range $[2,3]$. In particular, thanks to the exceptional capabilities of its PACS [4] and SPIRE [5] cameras to recover dust thermal emission from cold compact bright sources as well as diffuse and extended clouds and filaments $[2,6,7,8,3,9,10]$, Hi-GAL has delivered a new and more complete view of the evolutionary path that brings cold and diffuse interstellar material to condense into clouds and filaments that fragment into protocluster-forming dense clumps.

In this paper, we briefly present some of the main aspects and results of the statistical analysis of Hi-GAL compact sources. Such objects, which (depending on their heliocentric distance) can be classified as cores, clumps (the most numerous category), or even entire clouds, represent the places of Milky Way in which the earliest phases of star formation are expected to be occurring, or to start in the future. The five-band photometry of Hi-GAL compact sources was presented in [3] for the inner Galaxy $\left(-71^{\circ}<\ell<67^{\circ}\right)$, and the derivation of the physical parameters for sources provided with a reliable spectral energy distribution (SED) was discussed by [10], while the corresponding analysis (about both the photometric and physical parameters) for the outer Galaxy is still ongoing, with the exception of a portion between longitudes $216 .{ }^{\circ} 5$ and $225 .{ }^{\circ} 5$, already studied by [7].

In Section 2 a brief summary of map making and source extraction is provided. In Section 3 we describe how source physical properties were derived from photometry. In Sections 4 and 5 we show how these quantities can be used to characterize the Hi-GAL sources from the point of view of gravitational stability and evolutionary status, respectively. Finally, in Section 6 we give our conclusions.

\section{Map making, source extraction and selection}

The Hi-GAL photometric maps have been produced with the ROMAGAL pipeline [11] for the inner Galaxy portion, and with the UNIMAP pipeline [12] for the outer Galaxy, respectively. These algorithms optimally capitalize on the excellent sensitivity and stability of the bolometer arrays of the Herschel PACS and SPIRE photometric cameras, to deliver images of exquisite quality and dynamical range, absolutely calibrated with Planck and IRAS, and recovering extended emission at 
all wavelengths and all spatial scales. An example of RGB composite Hi-GAL mosaic is provided in Figure 1.

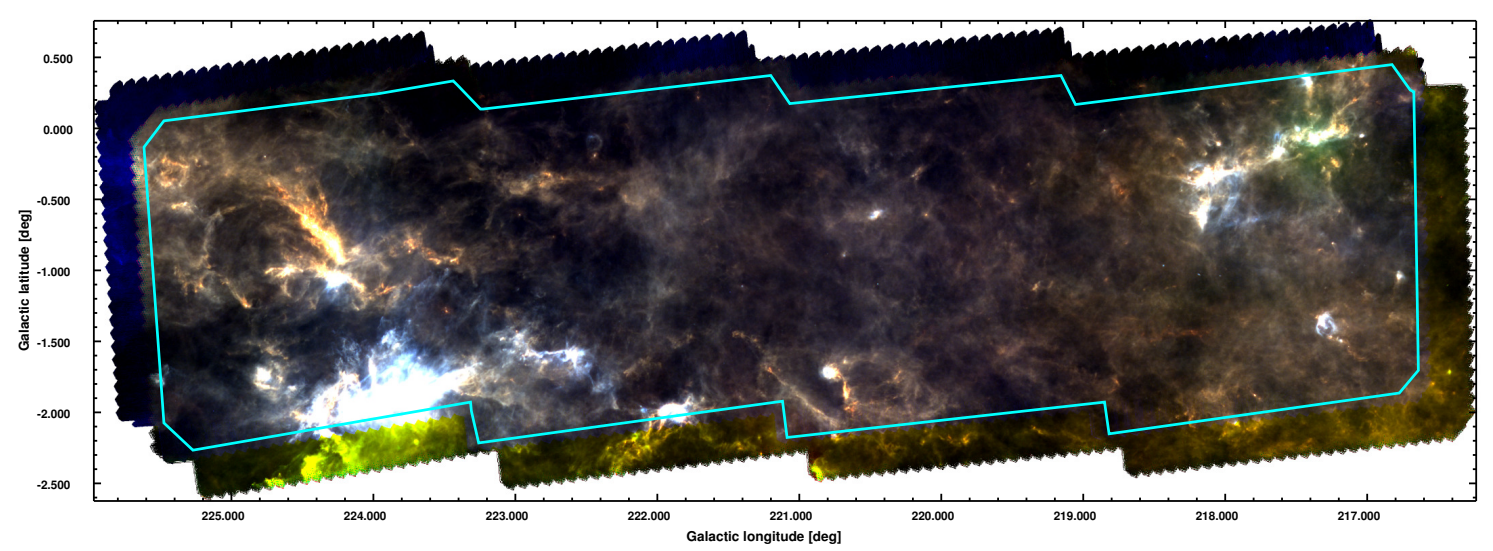

Figure 1: Example of Hi-GAL maps: RGB composite of the $216 .^{\circ} 5<\ell<225 .^{\circ} 5 \mathrm{Hi}-\mathrm{GAL}$ field (blue: PACS $160 \mu \mathrm{m}$; green: SPIRE $250 \mu \mathrm{m}$; red: SPIRE $500 \mu \mathrm{m}$ ), from [7]. The color scaling is linear. The cyan contour delimits the common science area covered by both PACS and SPIRE.

The compact source catalogues have been generated with the CuTEx algorithm [13], specifically developed to optimize source detection and extraction in the extreme conditions of intense and spatially varying background that are found in the Galactic Plane in the thermal infrared. It is based on the study of the curvature of the images, evaluating the second derivative at any pixel of the image, then efficiently damping all emission varying on intermediate to large spatial scales, and amplifying emission concentrated in small scales. The final integrated fluxes are then estimated by CuTEx through a bi-dimensional Gaussian fit to the source profile.

The Hi-GAL single-band catalogues contain 141994, 322827, 355924, 215134, 110991 entries in the 70-, 160-, 250-, 350- and 500- $\mu \mathrm{m}$ bands, respectively. The locations of these sources in Galactic coordinates are shown in Figure 2. The subsequent operation of band merging is based on iterating a positional matching $[6,7,10]$. Through this process, a catalogue of 938705 SEDs is produced, in which each entry can contain from one to five detections in as many bands.

At this point, it is needed to filter this five-band catalogue, in order to identify SEDs that are eligible for the modified black body (hereafter grey body) fit, hence to derive the physical properties of the objects. This selection is based on considerations about the regularity of the SEDs in the 160$500 \mu \mathrm{m}$ range, since the $70 \mu \mathrm{m}$ band is generally expected to depart from a grey-body behaviour (e.g., $[14,15])$. In this respect, only sources belonging to the common PACS+SPIRE area and detected at least in three consecutive Herschel bands were selected. Subsequently, we searched for further irregularities in the SEDs such as dips in the middle, or peaks at $500 \mu \mathrm{m}$. Further detail can be found in [10]. At the end of this filtering pipeline, we remain with 150223 sources, 100922 of which lie in the inner Galaxy, according to the definition given in Section 1.

Finally, a first and coarse classification is made between protostellar sources, expected to host already star formation activity, and starless ones, based on the presence or the lack of a detection at $70 \mu \mathrm{m}$. In Figure 3 an example of spatial distribution of "filtered" sources in a portion of the outer Galaxy is provided. 


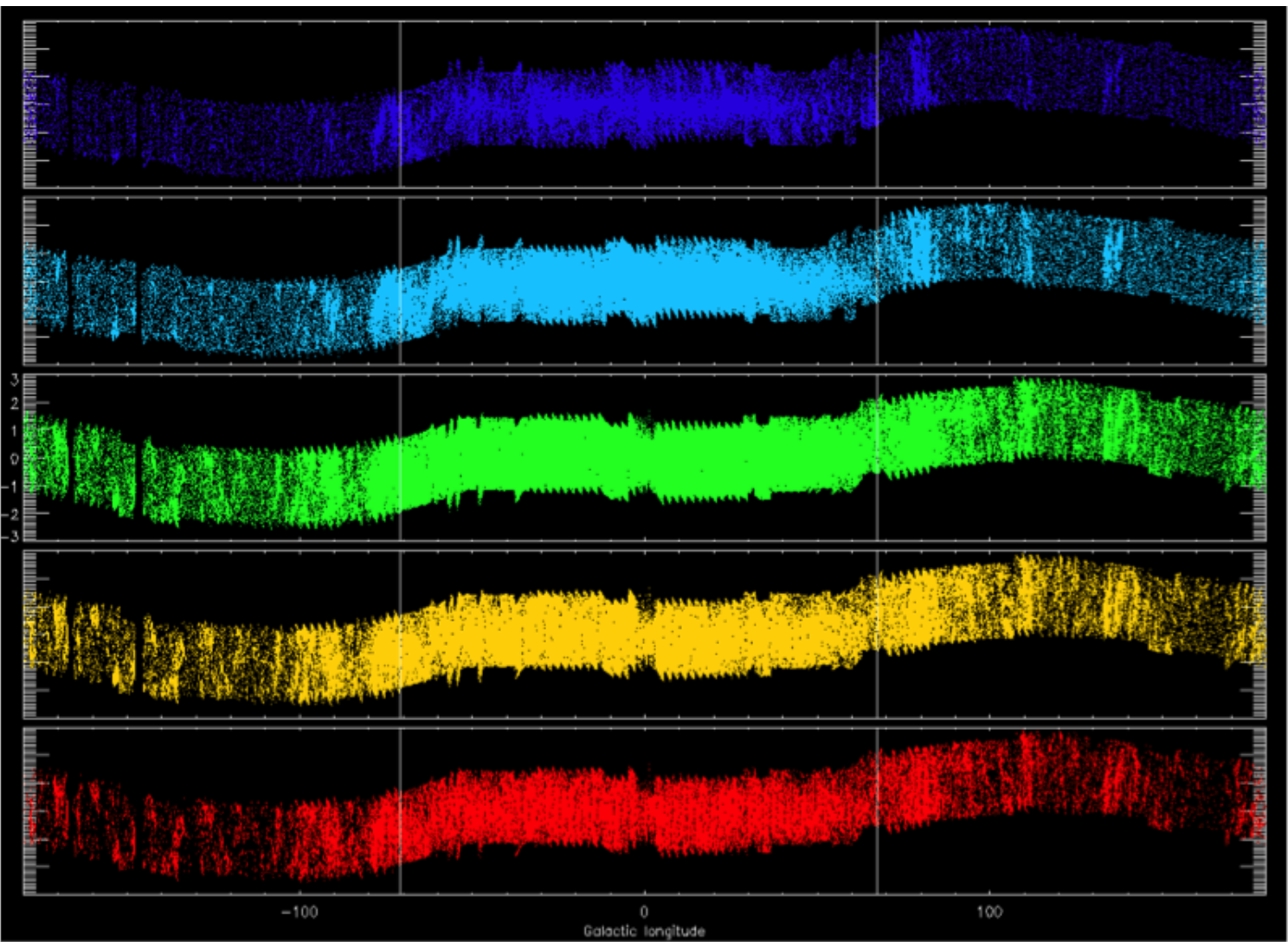

Figure 2: Distribution in the $[\ell, b]$ plane of the Hi-GAL compact sources detected in single bands by [3]. From top to bottom: $70 \mu \mathrm{m}$ (blue dots), $160 \mu \mathrm{m}$ (cyan), $250 \mu \mathrm{m}$ (green), $350 \mu \mathrm{m}$ (yellow), $500 \mu \mathrm{m}$ (red).

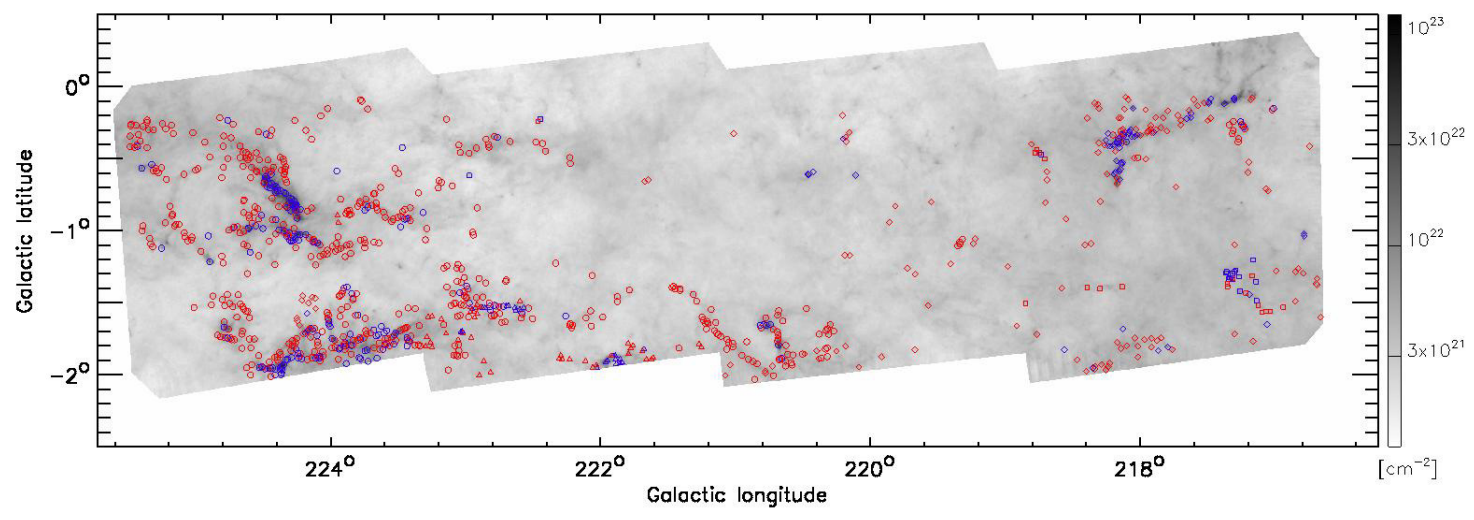

Figure 3: Spatial distribution of the Hi-GAL compact sources (surviving the filtering of the band-merged catalogue), overplotted on the column density map of the same field shown in Figure 1 (details about the derivation of the column density map can be found in [7]). The blue and red colors indicate protostellar and starless sources, respectively. 


\section{Derivation of source physical properties}

For every entry in the band-merged filtered catalogue, we searched for counterparts at $24 \mu \mathrm{m}$ (MIPSGAL, [16]), as well as at $21 \mu \mathrm{m}$ (MSX, [17]) and $22 \mu \mathrm{m}$ (WISE, [18]). The fluxes of these counterparts, typically associated with a warm internal component of the clump, are not considered for subsequent grey-body fitting, which is limited to the portion of the SED produced by cold dust emission, but only for estimating the source bolometric luminosity. In particular, since the choice of sources in the catalogue of [16] is rather conservative, we performed an additional search of sources in the MIPSGAL maps, using the APEX and DAOFIND source extractors in order to recover those sources that, from a visual inspection, appear to be real but were not included in the original catalogue. In this way, approximately 2000 additional SEDs were complemented with a flux at $24 \mu \mathrm{m}$.

On the other side of the SED, at wavelengths longer than $500 \mu \mathrm{m}$, we cross-matched our band-merged and filtered catalogue with those of [19] for the ATLASGAL survey (870 $\mu \mathrm{m},[20])$ and of [21] for the BOLOCAM Galactic Plane Survey (1100 $\mu \mathrm{m}$, [22]), respectively. If a match was found, these fluxes were involved in the subsequent grey body fit.

To derive distance-dependent quantities such as physical size, mass, and bolometric luminosity, we needed to compute heliocentric distances for our sources, since this information was available only for a limited number of known objects, and did not exist for the majority of Hi-GAL sources. We adopted the scheme presented in Russeil et al. (2011) to assign kinematic distances from ${ }^{12} \mathrm{CO}{ }^{13} \mathrm{CO}$ spectra from the FCRAO Galactic Ring Survey, the Exeter-FCRAO Survey, and the NANTEN survey. Out of the 100922 sources of the band-merged filtered catalogue of the inner Galaxy of [10], the determination of the heliocentric distance was successful in 57065 cases, i.e. $56 \%$.

A single grey-body was fitted, according to Equation 3 of [23], to the $\lambda>160 \mu$ m portion of the SEDs built as described above, to determine the average temperature $T$ of the source. The dust opacity exponent has been kept constant: $\beta=2$ (see Equation 4 of [23]). The mass was subsequently estimated through Equation 3 of [10]. SED fitting was performed by $\chi^{2}$ optimization of a grey-body model on a grid that is refined in successive iterations to converge on the final result. The strategy of generating an SED grid to be compared with data also gives us the advantage of applying PACS colour corrections directly to the model SEDs (since its temperature is known a priori for each of them), rather than correcting the data iteratively.

For sources with no assigned distance, the fit was performed anyway, to allow determination of distance-independent quantities (such as $T$ ).

The bolometric luminosity $L_{\mathrm{bol}}$ of the starless objects was estimated simply using the area under the best-fitting grey body, while for protostellar objects it was calculated by adding two contributions: the area under the best-fitting grey body starting from $160 \mu \mathrm{m}$ and longwards, plus the area of the observed SED between 21 and $160 \mu \mathrm{m}$ (for those counterparts detected in this range), to account for mid-infared emission contribution exceeding the grey body. Notice that the $L_{\mathrm{bol}} / M$ ratio is a distance-independent quantity. It is important for giving an evolutionary classification of sources, discussed in detail in Section 5. Other two distance-independent quantities have been derived considering both the observed SED and its grey body fit: the ratio between $L_{\mathrm{bol}}$ and its fraction $L_{\mathrm{smm}}$ due to the contribution of fluxes at sub-millimetre $(\lambda \geq 350 \mu \mathrm{m})$ wavelengths, 
and the bolometric temperature $T_{\mathrm{bol}}[24]$, defined as

$$
T_{\mathrm{bol}}=1.25 \times 10^{-11} \mathrm{~K} \times \frac{\int_{0}^{\infty} v F_{v} d v}{\int_{0}^{\infty} F_{v} d v},
$$

namely an estimate of the "average" frequency of the SED, weighted with fluxes and expressed in terms of a temperature. Indeed, for the SED of a perfect black body, $T$ and $T_{\text {bol }}$ coincide, while, for sources showing an excess in the SED at $\lambda \leq 70 \mu \mathrm{m}, T_{\text {bol }}$ is expected to increase significantly with respect to the temperature $T$ estimated at longer wavelenghts.

\section{Clump sizes, masses, and stability}

Our source population is distributed throughout the Galactic plane and in an extremely wide range of heliocentric distances, so that also the physical sizes of these compact objects (i.e. detected within a limited range of angular sizes) are expected to span a wide range of values. Linear sizes were derived only for objects provided with a distance estimate, starting from the angular size estimated bt CuTEx at the reference wavelength of $250 \mu \mathrm{m}$. According to the classification scheme proposed by [25] ( $r<0.1 \mathrm{pc}$ : cores; $0.1 \leq r \leq 1.5 \mathrm{pc}$ : clumps; $r>1.5 \mathrm{pc}$ : clouds) we find that only a small portion of the Hi-GAL compact sources are compatible with a core classification, while most of them are actually clumps, as it can be seen in Figure 4. Another very small fraction of sources, corresponding to the most distant cases, can be considered as entire clouds. Given the dominance of the clump-sized sources, hereafter it is practical to refer to the sources of the present work with the general term "clumps".

The underlying, generally inhomogeneous internal structure of these clumps is not resolved in our observations, but it can be reasonably supposed that they are composed by a certain number of cores and by an inter-core diffuse medium. Recently [29] demonstrated how cores detected in Herschel maps of nearby star forming regions $(d<0.5 \mathrm{kpc})$ get confused as the resolution of the maps is artificially deteriorated, simulating in this way an increase of the heliocentric distance of the observed region. With this technique they also explored the contribution of the inter-core emission to the total emission of the unresolved clumps, and the reliability of the clump global properties derived as in this work, checking to what extent they mirror the properties of the original population of the contained cores.

Figure 4 contains the mass vs radius plot for both the starless (left) and protostellar (right) clumps of our catalogue of inner Galaxy. Such a diagram can be used to illustrate and quantify the discussion of the gravitational stability of the starless clumps (neglecting the turbulence and magnetic field supports against gravity). Whereas the protostellar objects are expected to host ongoing star formation, among starless objects only gravitationally bound sources fulfill the conditions for a possible future collapse. Here we use the so-called Larson's third relation [26] to assess if an object can be considered bound:

$$
M(r)=460 M_{\odot}(r / \mathrm{pc})^{1.9},
$$

where $M$ is the source mass, and $r$ the radius. Masses above this threshold identify bound objects, i.e. genuine prestellar aggregates, while the remaining sources should be considered unbound objects. 


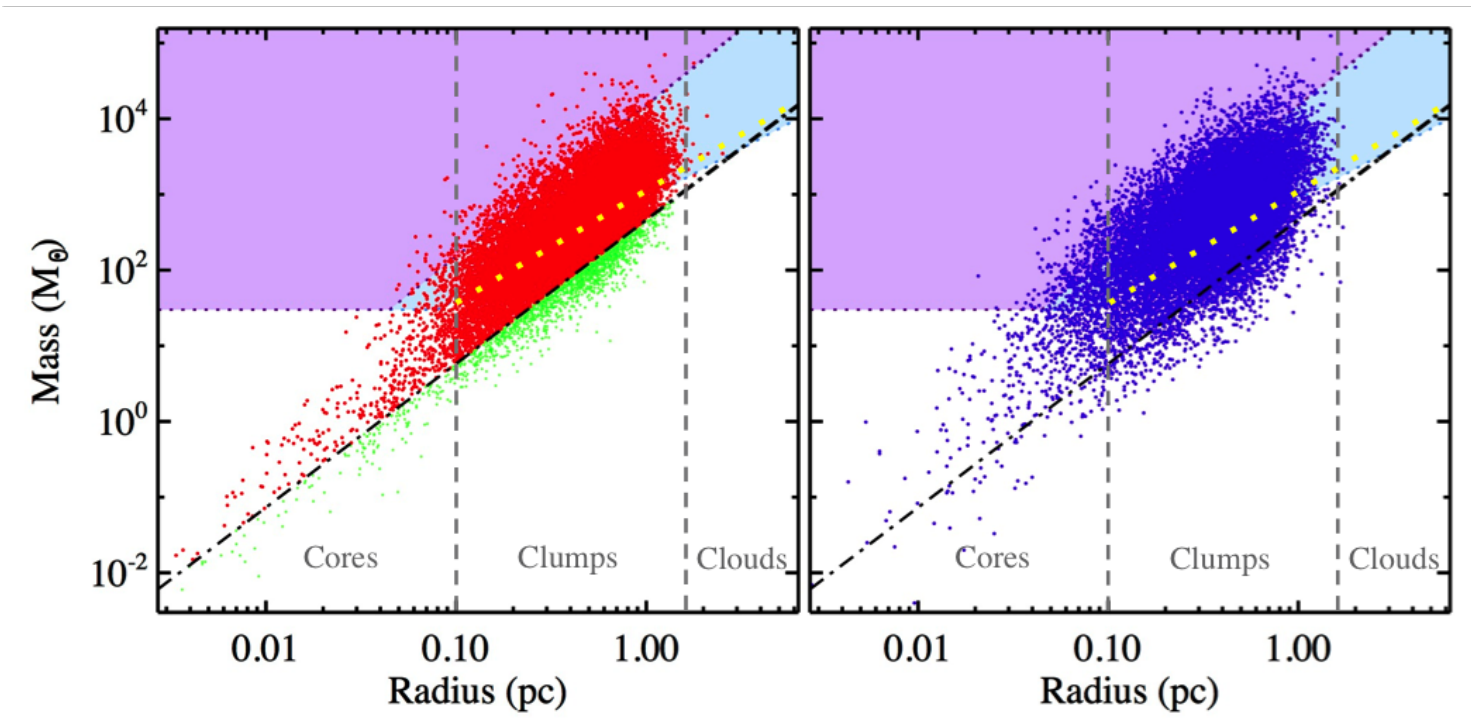

Figure 4: Left: mass versus radius plot for Hi-GAL starless sources. Prestellar (red) and unbound (green) sources are separated by the line $M(r)=460 M_{\odot}(r / \mathrm{pc})^{1.9}$ [26] (dot-dashed black line). The areas of the mass-radius plane corresponding to combinations fulfilling the thresholds of [27] and [28] for compatibility with high-mass star formation are filled with light blue and purple colors, respectively, the latter being contained in the former, and both delimited by a darker dotted line. Notice that adopting a lower limit of $10 M_{\odot}$ for the definition of a massive star, and a star formation efficiency factor of $1 / 3$ for the core-to-star mass transfer as in [7], these zones cannot extend below $30 M_{\odot}$. Additionally, the threshold of [29] is overplotted as a yellow dotted line. Finally, the critical radius values for the core/clump/cloud classification of [25] are plotted as vertical grey dashed lines. Right: the same as in left-hand panel, but for protostellar sources.

Through the mass vs radius this plot, moreover, it is possible to discriminate if a given source fulfills the necessary (but not sufficient) condition for massive star formation to occur, as determined by [28] in terms of surface density: $\Sigma_{\text {crit }}=1 \mathrm{~g} \mathrm{~cm}^{2}$ (that is to say $M(r) \propto r^{2}$ ). However, [27], based on empirical arguments, proposed a less demanding threshold, namely $M(r)>$ $870 M_{\odot}(r / \mathrm{pc})^{1.33}$ as a minimum condition for massive star formation. Finally, [29], discussing the distance bias affecting the source classification based on the two aforementioned thresholds, discovered that, as a general trend, an increasing distance of the sources clears out the zone of [28] and at the same time fills the one of [27], suggesting that the "true" mass threshold for compatibility with massive star formation should be a power law with an intermediate slope between these two. Minimizing false positives fulfilling the massive star formation threshold at increasing distances, [29] elaborated their own criterion: $M(r)>1282 M_{\odot}(r / \mathrm{pc})^{1.42}$. A relevant number of starless sources appear to be compatible with massive star formation based on the three thresholds: 12431 with respect to [27], 9973 to [29], and 546 to [28], respectively. This translates into an interestingly large sample of targets for subsequent study of the initial conditions for massive star formation throughout the Galactic plane.

Rigorously speaking, however, such considerations about the initial conditions for massive star formation should be applied only to the prestellar clumps, since in the protostellar ones a non- 
negligible part of the initial mass has already been transferred on to the forming star(s) or dissipated under the action of stellar radiation pressure or through jet ejection. However, the presence of a large number of protostellar clumps with large masses and relatively limited sizes (Figure 4, right panel) suggests anyway thousands of candidates for further study of early phases of ongoing massive star formation.

\section{Evolutionary descriptors}

The relation between bolometric luminosity and envelope mass is particularly interesting as an indicator of the evolutionary status of a core/clump. The $L_{\mathrm{bol}}$ versus $M_{\mathrm{env}}$ diagram is a widely used tool $[30,31,32,10]$ in which evolutionary tracks, essentially composed of an accretion phase and a clean-up phase [31,33], can be plotted and compared with data. In the earliest stages of star formation, as the protostar gains mass from the surrounding envelope, these tracks are nearly vertical, while, after the central star has reached the zero-age main sequence (ZAMS), they assume a nearly horizontal behaviour corresponding to dispersal of the residual clump material. Translating this behaviour in terms of $L_{\mathrm{bol}} / M$ ratio, we see an increase of this quantity both during the accretion and the clean-up phase (e.g., [6]), so that it is expected to overall increase with the age of the starforming clump.

In fact, studying in the longitude range $10^{\circ}<\ell<65^{\circ}$ the counterparts of the CORNISH survey sources [34, 35] considered as bona fide young HII regions, [36] obtained the distribution of the $L_{\mathrm{bol}} / M$ ratio of their Hi-GAL counterparts. The peak of this distribution lies around $L_{\mathrm{bol}} / M=$ $22.4 L_{\odot} / M_{\odot}$. At values larger than this peak, in principle, even more evolved sources are expected, so the fact that the drop of the distribution of [36] beyond this peak is due to lack of completeness, because the SED filtering we apply (mostly based on the availability of detections at 250 and $350 \mu \mathrm{m}$ ) causes the removal of a large number of evolved sources from our physical catalogue. Since our catalogue covers an area of the sky larger than that observed by the CORNISH survey to date, we adopted this Herschel-based method to identify possible HII candidates in other parts of the Galactic plane, such as the the fourth quadrant: the protostellar sources with $L_{\mathrm{bol}} / M>$ $22.4 L_{\odot} / M_{\odot}$ will henceforth be treated as HII candidates.

A confirmation of the validity of this criterion comes from the analysis of the average temperatures of these sources, simply based on the grey-body fit of the long-wavelength portion of their SEDs, and which turns out to be definitely high in these cases. In Figure 5, the fraction of protostellar sources classified as HII-region candidates out of the total number of protostellar sources per bin of temperature is shown: at $T \gtrsim 26 \mathrm{~K}, 80 \%$ sources have an $L_{\mathrm{bol}} / M$ greater than the threshold required for classifying it as an HII-region candidate, while the $90 \%$ level is achieved at $T \gtrsim 28 \mathrm{~K}$. This is in good agreement with the estimate of $T=25 \mathrm{~K}$ for clumps hosting ultra-compact HIIregions given by [37].

As mentioned in Section 3, the $L_{\mathrm{bol}} / L_{\mathrm{smm}}$ ratio is another distance-independent evolutionary indicator, frequently discussed in the study of the low-mass star formation. It was used by [38] to characterize Class 0 objects [39], establishing a maximum threshold of 200 for identifying an object of this class. Subsequently, [40] refined this value to 100. Of course, Class 0/I/II/III nomenclature is meaningful only for single low-mass protostars, while in our study we address sources that do not correspond to single YSOs, and, furthermore, in a relevant fraction of cases, these might host 


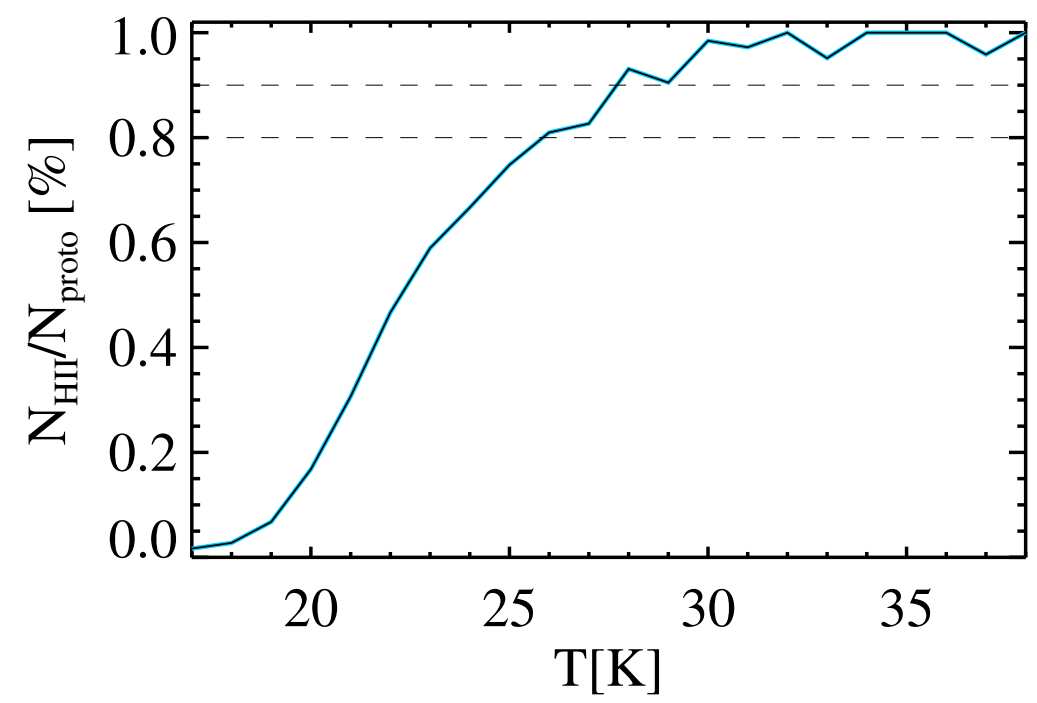

Figure 5: Fraction of protostellar sources classified as HII-region candidates (based on their $L_{\mathrm{bol}} / M$ ratio) over the total number of the protostellar ones vs temperature. The two horizontal dashed lines indicate the $80 \%$ and $90 \%$ levels.

massive star formation. Therefore, in our case, the $L_{\mathrm{bol}} / L_{\mathrm{smm}}$ ratio cannot be used to identify Class 0 sources (see also [41]), but we can safely state anyway that sources with a low $L_{\mathrm{bol}} / L_{\mathrm{smm}}$ ratio are at an early stage of star formation. We find that $99.96 \%$ of our prestellar sources are below the critical value of 100 , further confirming their primordial evolutionary status; instead, this fraction drops to $86 \%$ for protostellar sources. In particular, among protostellar clumps, almost the totality of the sub-population of HII-region candidates exceeds the threshold, confirming their nature of the most evolved objects in our catalogue.

Finally, also the distributions of the bolometric temperature $T_{\text {bol }}$ (introduced in Section 3) for the prestellar and protostellar sources are quite different in turn. Collecting the median values of the $T, L_{\mathrm{bol}} / M, L_{\mathrm{bol}} / L_{\mathrm{smm}}$, and $T_{\mathrm{bol}}$ for these two classes to build a metric, it is possible to see how different they appear from the evolutionary point of view. In Figure 6, left panel, we provide a synoptic view of the median values of these indicators, complemented with those of the source surface density $\Sigma$, reporting them on the five axes of a radar plot, in which points are connected through a polygonal line.

One can notice that the pentagon corresponding to the protostellar sources includes the pentagon representing the prestellar ones, since the values of the considered age indicators are typically larger for the former class of objects. The protostellar appear also, on average, more compact and dense (cf. also Figure 4) than prestellar ones. The same behaviour is also found for separated estimates of these parameters for the fourth and first Galactic quadrants, with no significant differences, except for a trend of evolutionary indicators to be slightly larger in the fourth quadrant.

This kind radar plot for the protostellar sources can be further compared with that of two extreme sub-samples of this class: the already discussed HII-region candidates, and, on the other side, the the MIR-dark protostellar sources, namely those detected at $70 \mu \mathrm{m}$, but not at mid infrared wavelengths, then expected to be among the earliest-stage objects inside the protostellar source population. In Figure 6, right panel, all evolutionary indicators appear decreased for the MIR- 

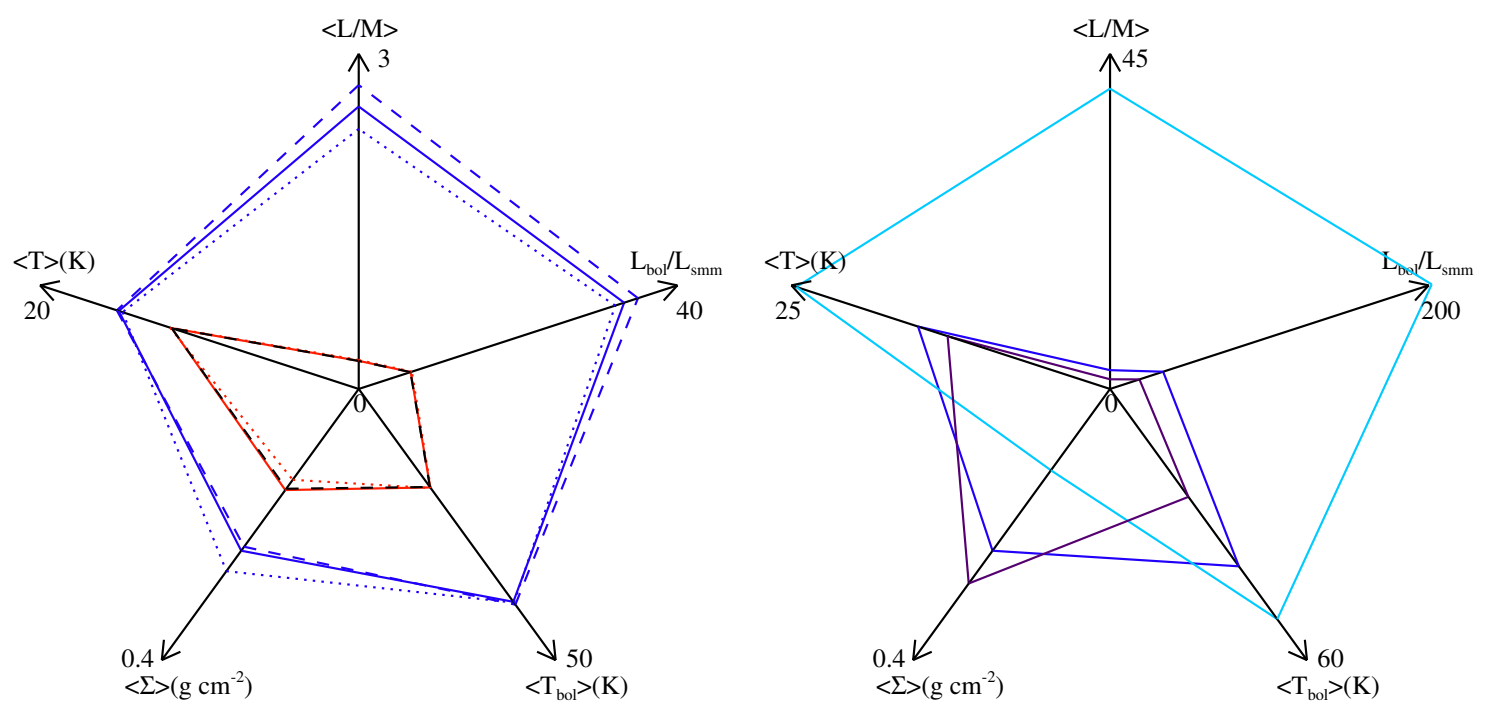

Figure 6: Left: radar plot for the median of five physical parameters (see text) for the classes of prestellar and protostellar clumps (red and blue lines, respectively). Solid lines represent the statistics of the entire populations, whereas separate statistics for the fourth and first Galactic quadrants are represented with dashed and dotted lines, respectively. Scales on each axis are linear, ranging from 0 to the value specified at the end. Right: as in the left panel, but for protostellar sources (blue line) and for the two extracted populations of MIR-dark sources (dark-purple line) and HII-region candidates (light-blue line), respectively

dark sample, and enhanced for the HII-region candidates, with respect to the overall protostellar population, except for the surface density, which exhibits the opposite global trend in the case of HII-regions. In this respect, an evolutionary sequence through various classes might be identified also for $\Sigma$ : MIR-dark sources are typically denser than prestellar sources, but also denser than generic protostellar ones; finally, on average HII-region candidates are found at lower densities than the generic protostellar sources (probably compatible with the clean-up phase testified by their high $L_{\mathrm{bol}} / M$ ratio, as corroborated by [42] and [43]).

\section{Conclusions}

Hi-GAL is an unbiased survey of the Galactic plane at far-infrared/sub-mm wavelengths, aimed at studying the earliest phases of star formation through the observation of the dust continuum emission in compact aggregates, typically called cores or clumps (depending on their physical size).

From several hundred thousand sources of this kind detected independently at the five single Herschel wavebands in the Hi-GAL survey maps, we obtained a band-merged catalogue that, after selecting only SEDs eligible for grey-body fit, is composed by 150223 sources, 100922 of which are located in the inner Galaxy [10], and, in particular, 24584 classified as having a protostellar content. For these 100922 sources we searched for ancillary photometry in the mid-infrared and in the sub-millimetre, managed to estimate the heliocentric distances in $56 \%$ of cases, and derived physical parameters through a grey-body fit. 
The large numbers involved in this analysis allowed us to build a meaningful statistics and to identify robust sub-samples of the main protostellar and prestellar source classes for future analysis of different star formation conditions and modalities across the Milky Way.

In particular, the mass vs radius plot allowed us to separate starless bound (prestellar) from unbound objects, and to identify, in the former class, clumps denoting compatibility with density conditions suitable for massive star formation (more than 50\% of prestellar clumps, according with the threshold of [29]).

The analysis of distance-independent parameters based only on Herschel photometry, such as $T, L_{\mathrm{bol}} / M, L_{\mathrm{bol}} / L_{\mathrm{smm}}, T_{\mathrm{bol}}$, and $\Sigma$ revealed a remarkable degree of segregation between the prestellar and protostellar clump population. The latter ones turn out to be, on average, warmer, denser, and more evolved than the former ones, confirming the initial classification based only on the presence or not of a detection at $70 \mu \mathrm{m}$.

Finally, we propose a criterion, based on a threshold on the Herschel-based $L_{\mathrm{bol}} / M$ ratio, for identifying HII-region candidates even in region of the Galactic plane not yet covered by dedicated radio surveys. These sources represent a sub-sample of the protostellar populations and are found to constitute the right tail of the distributions of all the aforementioned evolutionary descriptors.

In conclusion, the present Hi-GAL clump physical property catalog represents a huge reservoir of targets, already classified from the evolutionary point of view, for further specific analysis of massive star formation occurrences in the Milky Way.

\section{References}

[1] S. Molinari, B. Swinyard, J. Bally, M. Barlow, J.-P. Bernard, P. Martin et al., Hi-GAL: The Herschel Infrared Galactic Plane Survey, PASP 122 (2010) 314 [1001.2106].

[2] S. Molinari, B. Swinyard, J. Bally, M. Barlow, J.-P. Bernard, P. Martin et al., Clouds, filaments, and protostars: The Herschel Hi-GAL Milky Way, A\&A 518 (2010) L100 [1 005 . 3317].

[3] S. Molinari, E. Schisano, D. Elia, M. Pestalozzi, A. Traficante, S. Pezzuto et al., Hi-GAL, the Herschel infrared Galactic Plane Survey: photometric maps and compact source catalogues. First data release for the inner Milky Way: $+68^{\circ} \geq \ell \geq-70^{\circ}, A \& A 591$ (2016) A149 [1604.05911].

[4] A. Poglitsch, C. Waelkens, N. Geis, H. Feuchtgruber, B. Vandenbussche, L. Rodriguez et al., The Photodetector Array Camera and Spectrometer (PACS) on the Herschel Space Observatory, A\&A 518 (2010) L2 [1005 . 1487].

[5] M. J. Griffin, A. Abergel, A. Abreu, P. A. R. Ade, P. André, J.-L. Augueres et al., The Herschel-SPIRE instrument and its in-flight performance, A\&A 518 (2010) L3 [10 05 . 5123].

[6] D. Elia, E. Schisano, S. Molinari, T. Robitaille, D. Anglés-Alcázar, J. Bally et al., A Herschel study of YSO evolutionary stages and formation timelines in two fields of the Hi-GAL survey, A\&A $\mathbf{5 1 8}$ (2010) L97 [1005.1783].

[7] D. Elia, S. Molinari, Y. Fukui, E. Schisano, L. Olmi, M. Veneziani et al., The First Hi-GAL Observations of the Outer Galaxy: A Look at Star Formation in the Third Galactic Quadrant in the Longitude Range $216 .^{\circ} 5<\ell<225 .{ }^{\circ} 5$, ApJ 772 (2013) 45 [1304 . 7358].

[8] E. Schisano, K. L. J. Rygl, S. Molinari, G. Busquet, D. Elia, M. Pestalozzi et al., The Identification of Filaments on Far-infrared and Submillimiter Images: Morphology, Physical Conditions and Relation with Star Formation of Filamentary Structure, ApJ 791 (2014) 27 [1406.4443]. 
[9] M. Veneziani, E. Schisano, D. Elia, A. Noriega-Crespo, S. Carey, A. Di Giorgio et al., An analysis of star formation with Herschel in the Hi-GAL Survey. II. The tips of the Galactic bar, A\&A 599 (2017) A7 [1612.04995].

[10] D. Elia, S. Molinari, E. Schisano, M. Pestalozzi, S. Pezzuto, M. Merello et al., The Hi-GAL compact source catalogue - I. The physical properties of the clumps in the inner Galaxy $\left(-71.0^{\circ}<\ell<67.0^{\circ}\right)$, MNRAS 471 (2017) 100 [1706.01046].

[11] A. Traficante, L. Calzoletti, M. Veneziani, B. Ali, G. de Gasperis, A. M. di Giorgio et al., Data reduction pipeline for the Hi-GAL survey, MNRAS 416 (2011) 2932 [11 06.0698 ].

[12] L. Piazzo, L. Calzoletti, F. Faustini, M. Pestalozzi, S. Pezzuto, D. Elia et al., UNIMAP: a generalized least-squares map maker for Herschel data, MNRAS 447 (2015) 1471.

[13] S. Molinari, E. Schisano, F. Faustini, M. Pestalozzi, A. M. di Giorgio and S. Liu, Source extraction and photometry for the far-infrared and sub-millimeter continuum in the presence of complex backgrounds, A\&A 530 (2011) A133 [1011.3946].

[14] S. Bontemps, P. André, V. Könyves, A. Men'shchikov, N. Schneider, A. Maury et al., The Herschel first look at protostars in the Aquila rift, A\&A 518 (2010) L85 [1 005.2634 ].

[15] N. Schneider, T. Csengeri, M. Hennemann, F. Motte, P. Didelon, C. Federrath et al., Cluster-formation in the Rosette molecular cloud at the junctions of filaments, A\&A 540 (2012) L11 [1203.6472].

[16] R. A. Gutermuth and M. Heyer, A $24 \mu \mathrm{m}$ Point Source Catalog of the Galactic Plane from Spitzer/MIPSGAL, AJ 149 (2015) 64 [1412.4751].

[17] M. P. Egan, S. D. Price, K. E. Kraemer, D. R. Mizuno, S. J. Carey, C. O. Wright et al., VizieR Online Data Catalog: MSX6C Infrared Point Source Catalog. The Midcourse Space Experiment Point Source Catalog Version 2.3 (October 2003), VizieR Online Data Catalog 5114 (2003).

[18] E. L. Wright, P. R. M. Eisenhardt, A. K. Mainzer, M. E. Ressler, R. M. Cutri, T. Jarrett et al., The Wide-field Infrared Survey Explorer (WISE): Mission Description and Initial On-orbit Performance, AJ 140 (2010) 1868 [1008.0031].

[19] T. Csengeri, J. S. Urquhart, F. Schuller, F. Motte, S. Bontemps, F. Wyrowski et al., The ATLASGAL survey: a catalog of dust condensations in the Galactic plane, A\&A 565 (2014) A75 [1312. 0937 ].

[20] F. Schuller, K. M. Menten, Y. Contreras, F. Wyrowski, P. Schilke, L. Bronfman et al., ATLASGAL The APEX telescope large area survey of the galaxy at $870 \mu \mathrm{m}, A \& A \mathbf{5 0 4}$ (2009) 415 [0903.1369].

[21] A. Ginsburg, J. Glenn, E. Rosolowsky, T. P. Ellsworth-Bowers, C. Battersby, M. Dunham et al., The Bolocam Galactic Plane Survey. IX. Data Release 2 and Outer Galaxy Extension, ApJS 208 (2013) 14 [1305.6622].

[22] E. Rosolowsky, M. K. Dunham, A. Ginsburg, E. T. Bradley, J. Aguirre, J. Bally et al., The Bolocam Galactic Plane Survey. II. Catalog of the Image Data, ApJS 188 (2010) 123 [0909.2871].

[23] D. Elia and S. Pezzuto, Remarkable analytic relations among greybody parameters, MNRAS 461 (2016) 1328 [1606.02496].

[24] P. C. Myers and E. F. Ladd, Bolometric temperatures of young stellar objects, ApJ 413 (1993) L47.

[25] E. A. Bergin and M. Tafalla, Cold Dark Clouds: The Initial Conditions for Star Formation, ARA\&A 45 (2007) 339 [0705.3765].

[26] R. B. Larson, Turbulence and star formation in molecular clouds, MNRAS 194 (1981) 809. 
[27] J. Kauffmann and T. Pillai, How Many Infrared Dark Clouds Can form Massive Stars and Clusters?, ApJ 723 (2010) L7 [1009.1617].

[28] M. R. Krumholz and C. F. McKee, A minimum column density of $1 \mathrm{~g} \mathrm{~cm}^{-2}$ for massive star formation, Nature 451 (2008) 1082 [0801.0442].

[29] A. Baldeschi, D. Elia, S. Molinari, S. Pezzuto, E. Schisano, M. Gatti et al., Distance biases in the estimation of the physical properties of Hi-GAL compact sources - I. Clump properties and the identification of high-mass star-forming candidates, MNRAS 466 (2017) 3682 [1701.08035].

[30] P. Saraceno, P. Andre, C. Ceccarelli, M. Griffin and S. Molinari, An evolutionary diagram for young stellar objects., A\&A 309 (1996) 827.

[31] S. Molinari, S. Pezzuto, R. Cesaroni, J. Brand, F. Faustini and L. Testi, The evolution of the spectral energy distribution in massive young stellar objects, A\&A 481 (2008) 345.

[32] T. Giannini, D. Elia, D. Lorenzetti, S. Molinari, F. Motte, E. Schisano et al., The Herschel view of the on-going star formation in the Vela-C molecular cloud, A\&A 539 (2012) A156 [1202.1413].

[33] M. D. Smith, Evolutionary tracks of massive stars during formation, MNRAS 438 (2014) 1051 [1311.3352].

[34] M. G. Hoare, C. R. Purcell, E. B. Churchwell, P. Diamond, W. D. Cotton, C. J. Chandler et al., The Coordinated Radio and Infrared Survey for High-Mass Star Formation (The CORNISH Survey). I. Survey Design, PASP 124 (2012) 939 [1208.3351].

[35] C. R. Purcell, M. G. Hoare, W. D. Cotton, S. L. Lumsden, J. S. Urquhart, C. Chandler et al., The Coordinated Radio and Infrared Survey for High-mass Star Formation. II. Source Catalog, ApJS 205 (2013) 1 [1211. 7116].

[36] R. Cesaroni, M. Pestalozzi, M. T. Beltrán, M. G. Hoare, S. Molinari, L. Olmi et al., Infrared emission of young HII regions: a Herschel/Hi-GAL study, A\&A 579 (2015) A71.

[37] P. Hofner, F. Wyrowski, C. M. Walmsley and E. Churchwell, A $C^{17}$ O Survey toward Ultracompact H II Regions, ApJ 536 (2000) 393.

[38] P. André, D. Ward-Thompson and M. Barsony, From Prestellar Cores to Protostars: the Initial Conditions of Star Formation, Protostars and Planets IV (2000) 59 [arXiv:astro-ph/9903284].

[39] P. Andre, D. Ward-Thompson and M. Barsony, Submillimeter continuum observations of Rho Ophiuchi A - The candidate protostar VLA 1623 and prestellar clumps, ApJ 406 (1993) 122.

[40] A. J. Maury, P. André, A. Men'shchikov, V. Könyves and S. Bontemps, The formation of active protoclusters in the Aquila rift: a millimeter continuum view, A\&A 535 (2011) A77 [1108.0668].

[41] C. Fallscheer, M. A. Reid, J. Di Francesco, P. G. Martin, T. Hill, M. Hennemann et al., Herschel Reveals Massive Cold Clumps in NGC 7538, ApJ 773 (2013) 102 [1307.0022].

[42] A. Giannetti, J. Brand, Á. Sánchez-Monge, F. Fontani, R. Cesaroni, M. T. Beltrán et al., Physical properties of high-mass clumps in different stages of evolution, A\&A 556 (2013) A16 [1307. 4932].

[43] A. E. Guzmán, P. Sanhueza, Y. Contreras, H. A. Smith, J. M. Jackson, S. Hoq et al., Far-infrared Dust Temperatures and Column Densities of the MALT90 Molecular Clump Sample, ApJ 815 (2015) 130 [1511.00762]. 